\title{
Cognition in action: imaging brain/body dynamics in mobile humans
}

\author{
Klaus Gramann ${ }^{1,4, *}$, Joseph T. Gwin ${ }^{2}$, \\ Daniel P. Ferris ${ }^{2}$, Kelvin Oie ${ }^{3}$, Tzyy-Ping Jung ${ }^{1,4}$, \\ Chin-Teng Lin ${ }^{4,5}$, Lun-De Liao ${ }^{4,5}$ and Scott Makeig ${ }^{1}$ \\ ${ }^{1}$ Swartz Center for Computational Neuroscience, Institute for \\ Neural Computation, University of California, San Diego, \\ CA, USA \\ ${ }^{2}$ Human Neuromechanics Laboratory, School of Kinesiology, \\ University of Michigan, Ann Arbor, MI, USA \\ ${ }^{3}$ Translational Neuroscience Branch, Human Research and \\ Engineering Directorate, US Army Research Laboratory, \\ Aberdeen Proving Ground, MD, USA \\ ${ }^{4}$ Brain Research Center, National Chiao Tung University, \\ Hsinchu, Taiwan \\ ${ }^{5}$ Department of Computer Science, National Chiao Tung \\ University, Hsinchu, Taiwan \\ *Corresponding author \\ e-mail: klaus@sccn.ucsd.edu
}

\begin{abstract}
We have recently developed a mobile brain imaging method (MoBI), that allows for simultaneous recording of brain and body dynamics of humans actively behaving in and interacting with their environment. A mobile imaging approach was needed to study cognitive processes that are inherently based on the use of human physical structure to obtain behavioral goals. This review gives examples of the tight coupling between human physical structure with cognitive processing and the role of supraspinal activity during control of human stance and locomotion. Existing brain imaging methods for actively behaving participants are described and new sensor technology allowing for mobile recordings of different behavioral states in humans is introduced. Finally, we review recent work demonstrating the feasibility of a MoBI system that was developed at the Swartz Center for Computational Neuroscience at the University of California, San Diego, demonstrating the range of behavior that can be investigated with this method.
\end{abstract}

Keywords: EEG; embodied cognition; independent component analysis (ICA); mobile brain imaging; wireless dry electrodes.

\section{Introduction}

\section{Imaging the human brain under real world task conditions}

The development of new brain imaging methods over the last century, starting with X-ray techniques in the first quarter of the 19th century (Fox, 1984), allowed for significant new insights into human brain function and pathology. With the rapid development of new imaging methods and increasingly powerful computer technology, additional brain imaging approaches developed, including positron emission tomography (PET) and functional magnetic resonance imaging (fMRI). In the year 2010 alone, 811 scientific publications using fMRI for cognition research were published (source: ISI citation index; 3184 for fMRI alone; 811 for fMRI and cognitive). All these studies investigated human brain activity of participants lying supine in noisy scanners to draw conclusions about the consequences of pathological states and the functional architecture of the human brain. The human brain, however, evolved to control and optimize behavior in an ever-changing environment and is thus inextricably linked to active exploration and human motor behavior in our surroundings (Wilson, 2002; Anderson, 2003). We often assume that brain activity measured in noisy scanners and dimly lit experimental cubicles, reflects a general principle of brain dynamics during cognitive processing. However, until recently, no study explicitly investigated whether the brain switches to a different modus operandi, while we actively behave, move, walk and orient in small- and largescale environments.

Recent results from single cell recordings in different species of behaving animals demonstrate different brain dynamic states dependent on the locomotor state of the animal. In Drosophila melanogaster, responses of motion-processing interneurons in the visual cortex double when the flies become airborne, as compared to when they are in a resting position (Maimon et al., 2010). This demonstrates that the gain of certain cell types changes with the locomotor state of the animal. A similar change in brain dynamic state was demonstrated in mice (Niell and Stryker, 2010), revealing that neurons in the mouse visual cortex, more than double their visually evoked firing rate, when the animal transitions from standing still to running. At the same time, spontaneous firing or stimulus selectivity of the cell does not change. These results support the assumption that changes in behavioral state are accompanied by changes in brain dynamic state to adapt to differences in incoming idiothetic and allothetic sensory information. While invasive recordings can measure brain dynamic states in stationary animals and recently also, in behaving animals, this approach is limited for research on healthy humans for ethical reasons. Electrocorticographic (ECoG) recordings in patients with intractable epileptic seizures undergoing presurgery monitoring can reveal important insights into human brain dynamics. However, this approach is restricted to a clinical population and participants have to remain stationary during the recordings. 
As a consequence, research on human subjects generally requires non-invasive methods, of which the most commonly used are fMRI and positron emission tomography $\left({ }^{18} \mathrm{~F}-\mathrm{FDG}\right.$ PET), which indirectly measure brain dynamics of human participants through cortical blood flow. Changes in blood flow are assumed to correlate with the metabolic needs of neural populations that are active during cognitive processing (Logothetis et al., 2001). These traditional brain imaging techniques provide excellent spatial resolution (ranging from 1 to $2 \mathrm{~mm}$ ) but have poor temporal resolution due to the sluggish nature of the hemodynamic response (on the order of seconds). More direct approaches to measure brain activity like electroencephalography (EEG) or magnetoencephalography (MEG), directly measure electrical activity of neural populations that are synchronously activated during cognitive processing. These methods provide excellent temporal resolution (in the range of milliseconds) but are traditionally not considered as imaging methods because of their relatively coarse spatial resolution (in the range of centimeters) and their historical focus on analysis of the signal in the time-domain.

Irrespective of their spatial or the temporal resolution, all methods described thus far require participants to remain motionless during signal acquisition. Due to the weight of fMRI, PET, and MEG sensors, these imaging methods are inherently stationary. While EEG sensors are light enough to wear during movement, traditional signal analysis approaches have not been able to handle the movement and muscle artifacts that contaminate these signals (Makeig et al., 2009). However, cognition evolved to control human behavior and is, therefore, based on our physical structure and uses the body to achieve behavioral goals (Wilson, 2002; Anderson, 2003; Makeig et al., 2009). Behavior is not only essential for humans to adapt to an ever-changing environment, but contributes to the neurogenesis of hippocampal neuropile (Kempermann et al., 2010). Locomotion is necessary to increase proliferation of hippocampal neuropile, while subsequent learning of newly acquired information is necessary to protect newly born neural matter from apoptosis. This suggests that exploration of new environments helps the proliferation of neural matter that then can be used to store new information that is needed for adapting to new environments.

Non-invasive recording of human brain activity during mobile behavior also has considerable potential to unlock information about spatial reasoning in the human brain. Human cognitive architecture have been successfully investigated for years using traditional imaging approaches, but open questions remain with respect to brain dynamics, underlying more natural and complex behaviors. One example is the investigation of cortical and sub-cortical activity associated with spatial orientation. More than a decade ago, several investigations provided impressive new insights into the neural foundations of spatial navigation in humans (Aguirre et al., 1998; Maguire et al., 1998; Gron et al., 2000). However, these studies had to neglect the influence of rotations and translations of the body during spatial orientation due to mechanical restrictions of the imaging method. Natural spatial orientation is accompanied by rich idiothetic information stemming from the vestibular and kinesthetic system used with each turn and each step, to automatically update egocentric spatial representations (Farrell and Robertson, 1998; Gramann, in press). The absence of natural idiothetic information during the orienting process is often associated with differences in individual strategies used to solve a spatial task (Gramann et al., 2005, 2006, 2010b). Only a mobile brain imaging method will allow for investigations of brain dynamics accompanying body rotations and translations associated with natural orienting behavior.

In addition to new insights into the complex interplay of active behavior and brain dynamics, non-invasive recordings of human brain dynamics during active behavior in humans may also have potential clinical benefits. Altered cortical activity accompanying physical impairments might be useful for diagnostics and training approaches to regain physical functions. It would improve our understanding of how humans coordinate complex motor plans and thus could improve research and treatment of neurological disabilities by allowing brain neuroplasticity to be monitored during rehabilitation (e.g., rehabilitation of gait function, Bakker et al., 2007; Boyd et al., 2007; Jahn and Zwergal, 2010). It could enable an electromechanical brain computer interface (BCI) to supplement impaired descending motor commands to increase treatment efficacy (Daly and Wolpaw, 2008), or it could facilitate neural control of prosthetic limbs or powered orthoses that provide motor augmentation or restore functional mobility to amputees and patients with paralysis (Hatsopoulos and Donoghue, 2009; Kim et al., 2009; Leuthardt et al., 2009; Scherberger, 2009).

This review will give examples of the tight coupling of the human physical structure with cognitive processing and the role of supraspinal activity during control of human stance and locomotion. Existing brain imaging methods for actively behaving participants are described and new sensor technology allowing for mobile recordings of different behavioral states in humans is introduced. Finally, we review recent work demonstrating the feasibility of a MoBI system that was developed at the Swartz Center for Computational Neuroscience at the University of California, San Diego, demonstrating the range of behavior that can be investigated with this method.

\section{Embodiment of cognitive processes}

Investigations on embodied cognitive processing support the assumption that the attributes, as well as the use of our physical structure, can significantly alter the dynamic brain processes underlying cognition. Modulation of brain dynamics can happen on a very short time-scale, as Casasanto and colleagues demonstrated in their study on motor action and emotional memory (Casasanto and Dijkstra, 2010). The authors showed that ostensibly meaningless actions were correlated with the emotional content of memories that people retrieve. By asking participants to place marbles in a bowl that was either located above or below their hands, while retrieving memories, the authors demonstrated that upward movements of the hand were correlated with increased retrieval of positive memories. In general, neurocognitive representations of embodied cognitive processes differ for people with 
systematic differences in body-dependent behavior [e.g., for, left- vs. right-handers whose understanding of action verbs correlates with activity in the premotor cortex contralateral to the dominant hand (Willems et al., 2010)].

Systematic differences in behaviors and underlying cognitive strategies will, over time, determine the accompanying functional brain dynamics and/or lead to structural changes based on cortical plasticity. Functional changes can be seen as changes in the extent and direction of activation or the functional reorganization of regional activations in different brain areas contributing to, e.g., motor skill learning (Doyon et al., 2002; Kelly and Garavan, 2005). Structural changes can be found, e.g., in taxi drivers with increasing hippocampus proper correlating with their experience in using a maplike representation of London (Maguire et al., 1998) or in expert string players demonstrating increased cortical representations of the fingers of the left hand (Elbert et al., 1995). These examples show that behavior plays a pivotal role in shaping the functional and structural basis of the human brain, as well as the influence of the human brain on behavior reflecting one of its most important functions: the optimization of behavior in an ever-changing environment. However, the restrictions of traditional imaging approaches thus far prohibited recordings of functional and structural dynamics while humans actively behaved. Over the last years, our group tackled the question of how brain dynamics can be measured in actively behaving participants using a mobile brain/body imaging method.

\section{Existing technologies for mobile brain imaging in humans}

\section{Near infrared spectroscopy}

Functional near-infrared spectroscopy (NIRS) can be used to image the human brain during mobile activities. NIRS uses transmission and reflectance of near infrared light to measure changes in blood hemoglobin concentrations. Based on the premise of neuro-vascular coupling, changes in blood hemoglobin concentrations are reflective of local metabolic neural activity. NIRS sensors are small and light enough to be mounted on the head during mobile activities. Researchers have used NIRS to demonstrate increases in oxygenated hemoglobin in the frontal, premotor, and supplementary motor cortex during walking (Miyai et al., 2001; Suzuki et al., 2004, 2008; Harada et al., 2009).

However, a central limitation of this technique is that hemoglobin concentration dynamics are slow, giving NIRS a temporal resolution on the order of several seconds (Irani et al., 2007). Thus, NIRS cannot be used to study the brain dynamics of goal-directed movements and fast embodied cognitive processes, which can be initiated and executed in fractions of a second. A mobile brain imaging approach has to have the temporal resolution allowing for investigating sub-second cognitive processes based on direct measures of brain activity. The only such method is the electroencephalogram (EEG) that uses sensors light enough to follow the participants' movements and directly measures electrical activity of the brain in the range of milliseconds.

\section{Electroencephalography}

EEG is the sensing and graphical display of electrical activity generated by the synchronous activity of millions of neurons in the brain by means of electrodes placed along the scalp (Nunez, 1981). EEG is a non-invasive tool that provides high temporal resolution and directly measures electrical brain activity as compared to more indirect measures of blood flow reflecting metabolic changes as a consequence of neuronal activity. EEG predominantly measures electrical signals originating in the neocortex. Because of the orthogonal orientation of pyramidal cells in the deeper layers of the neocortex with respect to the skull, electrical activity (excitatory and inhibitory post-synaptic potentials) of large populations of neurons produce far field potentials that can be recorded using electrodes placed on the participant's scalp. With appropriate amplification and sampling rate, the voltage differences between electrodes can be recorded up to several kilohertz.

While the EEG signal allows very high temporal resolution, it provides only coarse spatial resolution. This drawback of EEG is due to volume conduction and sensor locations outside the volume generating the signal. The human brain consists of biological tissue that allows transmission of electrical currents and thus can be considered a volume conductor. Electrical potentials spread from a current source, through the brain volume, towards the sensors located outside the brain. The capacitive component of the brain's biological tissue impedance and the electromagnetic propagation effect can be neglected for frequencies below $1 \mathrm{KHz}$. This allows for modeling the transmission of electrical currents in the brain with quasi-static Maxwell equations (Plonsey and Heppner, 1967). At any given time several sources will transmit currents through the brain volume, therefore, the scalp-recorded potential will be a linear mixture of all current sources. The traditional analytical approach of interpreting systematic changes of scalp-recorded potentials is thus confounded with the problem of an unknown number of synchronously active sources. In case of actively behaving participants, an unknown number of electrical current sources associated with eye movements, as well as activity from neck, cranial, and facial muscles, will contribute to the linear mixture of an unknown number of brain sources.

\section{Requirements for a new mobile brain imaging method}

\section{Requirements for mobile brain imaging sensor technology}

Traditional experimental setups use conventional wet $\mathrm{Ag} / \mathrm{AgCl}$ electrodes to measure EEG signals from the human scalp (Nunez, 1981; Winter et al., 2007). Their characteristics have been widely studied and discussed in detail, demonstrating excellent signal quality with proper skin preparation 
and the use of conductive gels (Thakor, 1999). However, the preparation of participants and the use of conductive gels to reduce skin-electrode interface impedance require time and are often associated with decrease of signal quality for a subset of electrodes (Ferree et al., 2001). Their use inevitably leaves residues on the scalp and preparation is generally time consuming and often uncomfortable because it involves abrasion of the outer skin layers. Repeated skin preparations and subsequent gel applications may also induce allergic reactions or infections. The use of wet electrodes is often associated with short circuits between two electrodes, in close proximity, when too much gel is applied (Roberto, 2010) and the signal quality may degrade over extensive time periods as the skin regenerates and/or the conductive gel dries (Ferree et al., 2001). Additionally, the use of gel is usually problematic in areas with dense hair volume, especially for long-term applications. Another important restriction of traditional wet electrodes is that they are hard-wired to the amplifier, via shielded or non-shielded cables. This is not a problem in the case of traditional laboratory experiments that require subjects to sit still. However, if EEG is used to analyze the brain dynamics of behaving subjects, cables will inevitably restrict participants' movements and introduce mechanical artifacts (e.g., cable sway) (Gramann et al., 2010a; Gwin et al., 2010).

\section{Dry wireless electrodes for mobile EEG acquisition To} overcome these restrictions and to improve performance of conventional wet electrodes, dry contact and non-contact type EEG electrodes have been developed (Chi et al., 2010). Dry contact electrodes can be separated into three categories including: 1) dry micro-electro-mechanical systems (MEMS) type electrodes, 2) dry fabric-based electrodes and 3) hybrid dry electrodes. Dry MEMS type electrodes include microneedles on their top surface used to penetrate the outer skin layers allowing for high quality signal acquisition on the forehead or other hairless sites (Griss et al., 2001, 2002; Ko et al., 2006; Ruffini et al., 2006). Ruffini et al. tested carbon nanotube (CNT)-based dry electrodes in human trials for the first time, demonstrating that dry MEMSbased electrodes can potentially be used for acquiring EEG signals without skin preparation or the use of conductive gels (Ruffini et al., 2006, 2008). However, the use of MEMSbased electrodes can be considered invasive and sometimes painful because parts of the electrodes penetrate the skin surface. In addition, dry MEMS electrodes can only be applied to the forehead or other nonhairy sites and have relative high manufacturing expense compared to other types of electrodes.

Recently, new fabric-based electrodes were developed allowing for non-invasive recordings of biopotential signals (Anna et al., 2007; Hoffmann and Ruff, 2007; Baek et al., 2008; Xu et al., 2008; Beckmann et al., 2010). Dependent on the specifications of the fabric, comparable performance of fabric-based electrodes and commercial $\mathrm{Ag} / \mathrm{AgCl}$ electrodes can be achieved for ECG applications (Beckmann et al., 2010). A recent study by Lin et al. successfully tested foambased electrodes covered with conductive fabric to record EEG signals from the human forehead (Lin et al., 2011).
Importantly, foam-based electrodes revealed decrease motion artifacts for actively behaving (e.g., walking) participants. Compared with MEMS-based electrodes, fabric-based electrodes are relatively comfortable, non-invasive, and less expensive. However, because of the reduction in the contact area of the skin-electrode interface caused by hair, fabricbased electrodes are less suitable to acquire signals from sites covered with hair.

In addition to MEMS-based and fabric-based electrode types, Matthews et al. proposed a hybrid dry electrode for EEG recordings (Matthews et al., 2007, 2008; Eric et al., 2009). This type of electrode combines high-impedance resistive capacitive characteristics, contacts the scalp surface without any skin preparation and is dependent on the high contact impedance between the scalp and electrode. However, these electrodes are composed of hard, inflexible materials, which can lead to discomfort or even pain when force is applied to the electrode. The inflexible material is also associated with significant signal distortions accompanying motion of the sensors (Chi et al., 2010). Moreover, the fabrication costs for high-contact impedance electrodes are higher than costs for other types of dry EEG electrodes. To overcome those drawbacks, Liao et al. proposed a novel dry spring contact probe EEG electrode for measuring EEG signals, especially over hairy sites (Liao et al., 2011), which is flexible and fits the scalp surface, even in case of movement or applied force from outside. The spring contact probes and thin plate act as a buffer to avoid pain and to support electrode-scalp fit; thus improving skin-electrode contact impedance during movement.

As an alternative to dry contact electrodes, non-contact (capacitive) electrodes have the potential to acquire EEG signals without direct contact to, or preparation of, the skin (Matthews et al., 2005; Fonseca et al., 2007). A major drawback of dry capacitive electrodes, however, is their sensitivity to motion artifacts and the necessity of designing new amplifiers to acquire signals from sensors with high source impedance (Chi et al., 2010).

\section{The future of sensor technology for mobile brain/body}

imaging Because of higher user comfort, as compared to traditional EEG systems and higher mobility for actively behaving participants, dry wireless sensors are essential for future mobile brain recordings (Chi et al., 2010). Many commercial EEG devices provide EEG technology for entertainment [e.g., Neurosky (San Jose, CA, USA), Emotiv (Hong Kong, ROC), StarLab (Barcelona, Spain), EmSense (San Francisco, CA, USA) and nia Game Controller (OCZ Technology, San Jose, CA, USA)] (Emotiv; Crowley et al., 2010) or monitoring sleeping status (MyZeo, Newton, MA, USA). The development of wireless portable EEG devices for use with dry electrodes has been rapidly evolving. Lin et al. developed a wearable EEG device (Mindo) for every day mobile use including a 4-channel foam-based electrode solution as well as a 16-channel spring contact probe solution (Lin et al., 2008). Chi et al. designed a wireless device for both EEG and ECG measuring with non-contact electrodes (Chi et al., 2009; Chi and Cauwenberghs, 2010). With the development of a proper readout circuit, devices with non- 
contact electrodes can be used to acquire signals through thin $\mathrm{t}$-shirts. There is little doubt that developing a real wearable and wireless EEG device based on dry/non-contact electrodes is an important trend that will influence future applications in a wide field of technologies and will allow for recording electrical brain activity in actively behaving participants. The development of adequate sensors to improve signal quality during active behavior and to reduce the time necessary to prepare participants, is an important prerequisite for the success of a new mobile brain/body imaging method.

\section{Requirements for mobile brain imaging software}

To develop a widely used brain imaging modality, it will also be necessary to develop software for performing joint analysis of the EEG and behavioral data - not a trivial task. Current efforts at the Swartz Center for Computational Neuroscience (SCCN), UCSD, address two basic issues.

First, an experimental real-time interactive control and analysis (ERICA) software framework, built on the DataRiver platform by Andrey Vankov and colleagues at SCCN, allows for collection and synchronization of concurrent data streams with different sampling rates (Delorme et al., 2011) (http:// sccn.ucsd.edu/wiki/DataSuite). The 'producer environment', developed by Dr. Vankov, allows for scripting of interactive experiments, in which subject stimulation and feedback can be interactively based on real-time analysis of the joint DataRiver data. Allied MatRiver and Enactor environments, by Nima Bigdeley-Shamlo and colleagues, allow real-time Matlab processing and visualization within the ERICA framework.

Second, the widely used EEGLAB environment for electrophysiological data analysis (Delorme and Makeig, 2004) (http://sccn.ucsd.edu/eeglab) has been broadened to allow analysis and visualization of multi-modal MoBI data via a Matlab toolbox, MoBILAB, under development by Alejandro Ojeda and colleagues at UCSD (http://sccn.ucsd.edu/wiki/ MoBILAB). An early target for MoBI analysis of EEG data is to identify the timing and nature of motor decision events via changes in body movement (e.g., movement starts and stops, or course alterations). These can be identified as local maxima in the jerk (third-derivative) magnitude time series of a subject's limb or motion capture marker trajectory. To identify these motion events, one must properly low-pass filter the motion capture data and then locate (and carefully check) local maxima in the jerk time series. MoBILAB now includes software for doing this. Once one or more classes of motor decision events are identified in the behavioral data, then standard analysis of EEG data epochs surrounding the events of interest may be performed using EEGLAB, either on the natural time-locked EEG epochs or after time warping the epochs to normalize the duration of one or more movement phases across epochs.

The development of adequate software to process the increasing amounts of synchronously recorded data and to enable scientist to explore the relationship of active behavior and brain dynamics is an important milestone for applying a mobile brain/body imaging approach.

\section{Requirements for analyzing mobile brain imaging data}

Traditional analysis of EEG and the problem of volume conduction Traditional cognitive experiments record EEG while subjects are seated in a dimly lit and sound attenuated room, waiting for stimuli to be presented, without moving any part of their body or even their eyes. Reactions to presented stimuli are usually restricted to single button presses or minimal movements of the feet or hands. The suppression of eye movements, or any other movements of the body, avoids the relatively strong electrical potentials that are associated with movement of the eye [the human eye can be considered a battery with a negatively charged retina (due to the negative membrane potential of receptor cells) and a relatively more positive cornea. Movement of the eyes to the sides or up and down cause strong electrical potentials that can be measured at electrodes far from the eyes] or contraction of neck or superficial skull and facial muscles (Makeig et al., 2009). Time periods with electromyographic or ocular activity are typically removed offline by rejecting the contaminated signal or by trying to regress out prototypic artifacts. The recorded signals are then epoched relative to the onset of a stimulus or class of stimuli and averaged over all epochs, assuming that activity unrelated to stimulus processing will be averaged out.

The restriction of participant movements in EEG investigations is thus primarily based on the fact that, due to volume conduction, nonbrain related activity will contaminate the signal of interest. However, movement of the eyes or contraction of muscles and the accompanying proprioceptive feedback reflect active cognition and impact information processing (Biguer et al., 1988; Bove et al., 2002; Hayhoe and Ballard, 2005).

Independent component analysis (ICA) for mobile brain imaging Spatial filter algorithms can be used to solve the problem of volume conduction and to dissociate brain from nonbrain related electrical activity. One example is independent component analysis (ICA), which separates multichannel data into independent component (IC) activities. Each IC activity is maximally statistically independent from any other IC activity and differs with respect to the strength of its volume-conducted activity at each sensor. Like other spatial filters, ICA is based on model assumptions including spatially stable mixtures of the activities of temporarily independent sources (brain and nonbrain), a linear summation of potentials arising from different areas, and a maximum number of independent sources being equal or less than the number of sensors. ICA can separate out activities of dozens of maximally independent information sources whose scalp maps nearperfectly fit the dipolar projections of cortical EEG sources (Makeig et al., 2002, 2004; Gramann et al., 2010b). As a consequence, equivalent current dipole models can be used without further model assumptions to locate the origin of the temporally-independent time source in the physical brain space. With respect to actively behaving participants, it 
should be pointed out that ICA separates out and dissociates the contribution of brain and nonbrain sources including mechanical artifacts (e.g., line noise), as well as biological signals that are important for cognitive processing [i.e., eye movements and muscle activities (Gramann et al., 2010a)].

\section{Cognition in action-imaging brain and nonbrain processes}

Recordings of electrical brain activity of mobile human participants are necessary to gain new insights into the brain dynamics accompanying natural embodied cognition. Only if information from all of the sensory systems involved in active behavior is available to the participant will natural cognitive processing be possible. This includes proprioception and vestibular inputs, as well as visual and auditory information during head turns or full body rotations and translations in space. However, MoBI recordings of human participants actively looking around in space, walking, pointing and reaching towards different objects will inevitably contain electromyographic (EMG) activity originating from superficial scalp muscles, extraocular muscles, neck muscles, and muscles contracting during mastication and swallowing.

The human neck comprises several ventral and dorsal muscles. The latter are used for rotation (looking to the side), flexion (looking down), hyperextension (looking up), and lateral flexion (ear to shoulder) of the head. The dorsal neck muscles consist of four layers with the outermost layer formed by the trapezius muscle, which does not significantly contribute to head motion (Bull et al., 1985; Vasavada et al., 2002). The principal muscles of the second layer are the splenius capitis (SPL) and levator scapulae (LS), while the principal muscles of the third and fourth layers are the semispinalis capitis (SSC) and the small muscles located between the occipital bone the cervical vertebrae, respectively. Contractions of all of these muscles, to move the head in space, show complex interactions with forces of gravity (MayouxBenhamou et al., 1997) and differential contributions to head movements along a combination of the cardinal axes. Most importantly, the complex and redundant anatomy of the neck musculature will result in cross talk of muscle activity recorded through surface EEG electrodes.

There are two main functions of muscles, to move the body and to stiffen the body. The purpose of most neck muscle activity is the latter. Opposing muscles hold our head erect at all times except during full supine relaxation. In addition, the neck musculature plays a central role in providing stability of the head for successful integration of sensory information during motion. As a result, neck muscle activity interacts with visual and auditory inputs, as well as proprioceptive and vestibular information (Peterson et al., 1985). Subtle postural position, shape, and tensional adjustments mold our readiness to perceive and to act - for instance when we lean forward to see more clearly, turn one ear forward to hear more clearly, or retract our head in fright. The complex neck and face muscular adjustments entailed in maintaining and adapting our erect posture to meet the challenge of events (including their accompanying expected opportunities and/or threats), are a key aspect of our emotional perception of and communication to others.

\section{Human stance control and imaging approaches}

Postural control has been studied in terms of two primary functional goals: postural equilibrium, the coordination of movement strategies to stabilize the body during both selfinitiated and externally-triggered movements that would disturb postural stability, as discussed in the previous section; and postural orientation, the maintenance of the body's alignment with respect to gravity, support surfaces, the visual environment, and internal reference frames, which largely comprises the control of upright stance. Historically, the neural control of postural behavior, in general, and stance control, in particular, has been thought to arise from brainstem and spinal circuits, with little consideration of the role of cortical mechanisms (Sherrington, 1910; Magnus, 1926). This view has been established by observations of intact postural control in animals with transections at the midbrain level (Magnus, 1926) and studies showing that early postural responses to mechanical perturbations occur more quickly than the fastest voluntary movement (Horak and Nashner, 1986; Inglis et al., 1994; Allum et al., 2001). For example, short forward or backward translations of the support surface, elicit stereotyped patterns of muscular activations, beginning around the ankle joint and proceeding temporally in a distal-to-proximal order, with an onset latency of approximately $100 \mathrm{~ms}$ (Horak and Nashner, 1986).

The view of postural control in terms of reflex control processes is consistent with classical views of motor performance in terms of formal control systems that minimize the role of cognitive processes in motor control (Cisek and Kalaska, 2010). Indeed, the postural system for upright, bipedal stance control has quite often been conceptualized as a sensorimotor feedback process (Morasso et al., 1999; Van der Kooij et al., 1999, 2001; Peterka, 2002; Mergner et al., 2003; Kiemel et al., 2006), whose time-varying output is reflected in trajectories of continuous deviations of body position within a limited spatial range (i.e., 'postural sway'). Numerous experimental results demonstrate the importance of sensory inputs for maintaining upright stance orientation relative to the physical environment, including vestibular (Johansson et al., 1995; Hlavacka et al., 1996), visual (Berthoz et al., 1979; Bardy et al., 1996), somatosensory (Aniss et al., 1992; Kavounoudias et al., 2001) and proprioceptive (Murphy et al., 2002) inputs. As such, the modeling of stance control as a feedback control process, has served as a useful model for examining processes of sensorimotor integration (Dijkstra et al., 1994) and multisensory processing (Van der Kooij et al., 1999; Jeka et al., 2000; Oie et al., 2002) in the nervous system.

Formal control theory models and system identification approaches, such as those mentioned above, have provided profound insights into the nature of the postural system itself and potential mechanisms for neural control. Still, how such control models are implemented in the nervous system is an 
open question. As we have discussed, the involvement of spinal, brainstem, and cerebellar circuits in postural control has been well demonstrated using decerebrate animal models (Sherrington, 1910; Magnus, 1926) and through clinical studies in humans (Bronstein et al., 1990; Horak and Diener, 1994; Lewko, 1996). More recent findings in animal models have demonstrated long-loop postural pathways through brainstem and motor cortex (Beloozerova et al., 2003, 2005) that are modified by multisensory inputs, including vision and vestibular. The output of these areas along descending pathways (i.e., reticulospinal, rubrospinal, corticospinal) is phasic, however, the relative contributions of these mechanisms compared to cerebellar, brainstem, and spinal influences is not yet clear.

While the potential role of long-loop postural responses in humans remains controversial (Jacobs and Horak, 2007), significant behavioral and clinical evidence indicates a role for the cortex in the neural control of posture, if only indirectly. For example, changing task demands (Stoffregen et al., 1999, 2000) and manipulations of cognitive load and attention (Carpenter et al., 2001; Hunter and Hoffman, 2001; Woollacott and Shumway-Cook, 2002; Quant et al., 2004) lead to decreases in postural stability. As well, patients with cortical lesions exhibit significant postural abnormalities (Diener et al., 1985; Perennou et al., 2000), and those with dementia or other cognitive deficits also increased postural instability and risk for falls (Elble and Leffler, 2000; Hauer et al., 2003) or reduced abilities to utilize sensory or mechanical aids (Sullivan et al., 2009).

Imaging of postural control Slobounov and colleagues recently reported cortical responses related to voluntary postural movements (Slobounov et al., 2005, 2008). Quant et al. have shown early (approx. $100 \mathrm{~ms}$ ) cortical responses that were evoked by unpredictable, external postural perturbations, and whose modulation by secondary task effects was associated with differences in postural response amplitude (Quant et al., 2004). However, the use of neuroimaging techniques in the study of human postural orientation has thus far been fairly limited. Recently, Slobounov et al. presented ICA-based source localization results that suggest anterior cingulate modulation of EEG in the $\vartheta, \alpha$, and $\gamma$ bands associated with changes in the virtual-time-to-contact of center-of-pressure position relative to postural stability boundaries (Slobounov et al., 2009). Such results, achieved through the joint analysis of the kinematic and brain dynamics, describing observed behavior illustrate the potential of MoBI-based brain imaging for elucidating the cortical implementation of putative mechanisms of predictive control, sensory estimation and/or sensory re-weighting that have been identified in previous mechanistic models of the postural control system.

Postural control and postural state may also serve as critical contextual variables for the interpretation of brain dynamics underlying human neurocognitive performance. For example, Caldwell and colleagues presented results showing that an upright standing vs. a seated posture significantly attenuated sleep-deprivation-induced increases in slow-wave EEG activity (e.g., absolute theta power), and that such postural manipulations mitigated sleepdeprivation-related decrements in cognitive performance (e.g., slower reaction time and increased attention lapses) (Caldwell et al., 2000, 2003). Similar postural changes have been interpreted in terms of homeostatic reflex mechanisms (Cole, 1989), while Tachtsidis and colleagues observed spontaneous, low frequency, cerebral hemodynamic oscillations whose amplitude is posture-dependent (supine vs. sitting vs. standing) (Tachtsidis et al., 2004). Along with previously discussed results indicating secondary task effects on postural control, such results indicate that it may be important to take into account postural dynamics and changes in postural state in understanding observed MoBIbased brain dynamics data.

Applications for clinical sciences As discussed above, the human control of stance has served as a useful model system for the study of sensorimotor integration and multisensory processing in the nervous system. The study of stance control has also been pursued for its practical and clinical implications; in the US, approximately 300000 falls result in hip fractures, with associated health care costs of approximately $\$ 10$ billion USD (Maki et al., 2003). The risk of injury from falling increases with age. Jeka and colleagues recently showed that both healthy and fall-prone older adults have higher gains and slower down-weighting in the face of high-amplitude visual environmental motion vs. young adult controls (Jeka et al., 2010). Understanding the neural underpinnings of sensory reweighting, including the potential role of cortical mechanisms may be pivotal in the design and enhancement of mitigation strategies based on sensory training. Moreover, cortical signatures that can be associated with predictive measures of postural instability (e.g., centerof-pressure time-to-contact with stability boundaries) may hold promise as the basis for the development of neutrally based assistive and/or training technologies for the prevention of falls (Slobounov et al., 2009).

\section{Human locomotor control and imaging approaches}

It is generally accepted that humans use a multifaceted locomotion control strategy, including descending (from the brain), peripheral (reflex pathways), and central neural networks (spinal) inputs (Dietz and Duysens, 2000; Dietz, 2003; Nielsen, 2003; Drew et al., 2004; Rossignol et al., 2006; Yang and Gorassini, 2006; Grillner et al., 2008). While spinal locomotor networks in humans and other vertebrates are capable of generating rhythmic muscle activity, activating these networks in humans without functional descending motor pathways has proven to be difficult (Dietz et al., 1995; Wirz et al., 2001; Ferris et al., 2004; Fong et al., 2009). Therefore, understanding how the brain, particularly the cortex, is involved in human locomotion is critical from a basic science perspective and for a variety of clinical applications.

In the absence of a method to image the human brain during locomotion, researchers have used a variety of creative approaches to study neural control of locomotion. One approach is transcranial magnetic stimulation 
(TMS). TMS can excite or suppress target areas of human cortex at precise phases of the gait cycle. Evaluating gait kinematics in response to these cortical stimulations has demonstrated substantial cortical involvement in locomotion (Schubert et al., 1997, 1999; Capaday et al., 1999; Christensen et al., 2001; Hiraoka and Abe, 2007; Iglesias et al., 2008). Another approach to study supraspinal contributions to human locomotion is to use stationary brain imaging modalities (e.g., fMRI and PET) while subjects perform rhythmic foot or leg movements (Luft et al., 2002; Dobkin et al., 2004; Sahyoun et al., 2004; Heuninckx et al., 2005; Heuninckx et al., 2008; Mehta et al., 2009) or mental imagery of locomotion (Jahn et al., 2004; Pfurtscheller et al., 2006; Jahn et al., 2008a; Jahn et al., 2008b; Wagner et al., 2008; Wang et al., 2008; Jahn et al., 2009; Ionta et al., 2010). However, these stationary tasks are fundamentally different than normal locomotion. Postural adjustments and navigation are not required during rhythmic leg movements. During mental imagery of walking, the sensory information going to the brain is substantially different than during actual walking (la Fougere et al., 2010). While recent studies using these methods have provided important insight into supraspinal control of human walking (Yang and Gorassini, 2006; Jahn and Zwergal, 2010), there is a clear need for a mobile technique to quantify brain activity. Early studies using the MoBI approach have demonstrated the feasibility of imaging electrocortical dynamics during human locomotion. Specifically, Gwin et al. (2011) recorded lower-limb kinematics, ground reaction forces and 256-channel EEG, while subjects walked on a treadmill.

Figure 1A shows the experimental set-up including infrared cameras and reflective markers on the lower limbs for motion capture, an instrumented in-ground treadmill for measuring ground reaction forces and a high-density EEG system. In this study, ICA parsed electrocortical source signals from EEG. Electrocortical sources in or near the anterior cingulate, posterior parietal and sensorimotor cortex exhibited significant intra-stride changes in spectral power
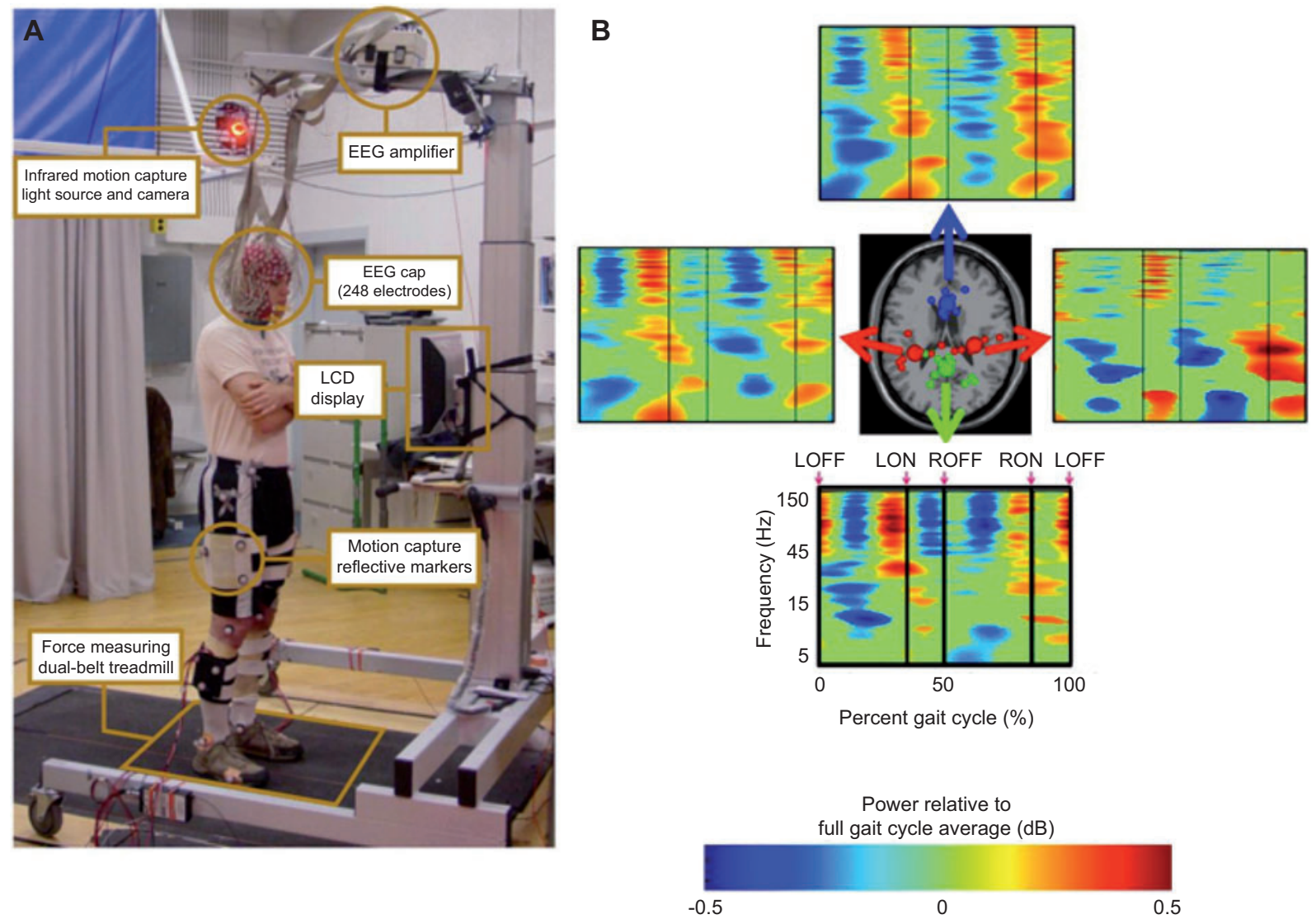

Figure 1 (A) A subject standing on the dual-belt treadmill facing the LCD display. Components of the experimental set-up are highlighted and described in the linked text boxes. (B) Gait event related spectral perturbation (ERSP) plots showing average changes in spectral power during the stride cycle relative to the full gait cycle baseline for (top) the anterior cingulate cluster, (bottom) the posterior parietal cluster, (left) the left sensorimotor cluster and (right) right sensorimotor cluster. The gait cycle begins and ends with left toe-off (LOFF). Vertical lines indicate the timing of left heel-strike (LON), right toe-off (ROFF), and right heel-strike (RON). Non-significant differences have been set to $0 \mathrm{~dB}$ (green). (Center) clusters of electrocortical sources localized to the anterior cingulate (blue), posterior parietal cortex (green), and sensorimotor cortex (red) are visualized in a horizontal view of the Montreal Neurological Institute (MNI) brain volume. Small spheres indicate the equivalent current dipole locations for single electrocortical sources for single subjects; larger spheres indicate geometric cluster centroids. 
(i.e., modulations in spectral power that were locked to distinct phases of the gait cycle) (Figure 1B). During the end of stance, as the leading foot was contacting the ground and the trailing foot was pushing off, $\alpha$ - and $\beta$-band spectral power increased in, or near, the left/right sensorimotor and dorsal anterior cingulate cortex. Power increases in the left/right sensorimotor cortex were more pronounced for contralateral limb push-off (ipsilateral heel-strike) than for ipsilateral limb push-off (contralateral heel-strike). Intrastride high- $\gamma$ spectral power changes were evident in anterior cingulate, posterior parietal and sensorimotor cortex.

Applications for clinical sciences There are many potential clinical applications for mobile brain imaging with EEG. Being able to non-invasively quantify how brain activation during gait is different in neurologically impaired patients compared to healthy individuals could help clinicians identify subsets of patients with similar symptoms and target interventions accordingly. These interventions may include rehabilitation from acute nervous system damage, such as stroke or spinal cord injury, as well as therapy for chronic nervous system degeneration, such as fall prevention techniques for older adults. To this end, we have begun to classify brain activation patterns associated with perturbed walking and walking under more demanding balance conditions. Freezing gait in patients with Parkinson's disease is another potential target. Being able to identify the electrocortical dynamics that cause individuals to become motionless without the ability to take a step could lead to new brain stimulation devices that prevent freezing gait from developing. Given the pervasiveness of gait disabilities across a wide variety of patient populations, the use of MoBI to understand the electrocortical dynamics associated with impaired gait is surely an avenue of inquiry worthy of pursuit.

Application for brain machine interfaces (BMI) The application of MoBI during locomotion could enable an electromechanical brain machine interface (BMI) to supplement impaired descending motor commands during gait rehabilitation, which might increase treatment efficacy (Daly and Wolpaw, 2008). Specifically, using features of brain activity to control electromechanical motor augmentation devices that compensate for impaired neuromuscular control during gait rehabilitation may increase the relearning rate by encouraging active neurological participation from patients and producing more normal sensory afferents that induce central nervous system plasticity.

In addition, MoBI could facilitate non-invasive neural control of prosthetic limbs or powered orthoses that provide motor augmentation or restore functional mobility to amputees and patients with paralysis (Millan et al., 2008; Hatsopoulos and Donoghue, 2009; Kim et al., 2009; Leuthardt et al., 2009; Scherberger, 2009). In vertebrate animals, intra-stride modulations of neuronal firing rates during walking, measured using implanted intra-cortical electrode arrays, have been accurately decoded to predict lower limb muscle activations and kinematics (Fitzsimmons et al.,
2009). More recently, Song and Giszter demonstrated that rats can adapt to a hind limb coupled BMI and utilize the BMI to overcome an imposed resistive load during locomotion (Song and Giszter, 2011). Future studies using noninvasive MoBI techniques and human subjects should examine motor adaptation to lower limb coupled BMIs, in particular BMIs designed to provide motor augmentation to overcome chronic gait impediments.

\section{Imaging cognitive processing during active locomotion}

In the first experiment investigating cognitive processes while participants walked on a treadmill, Gramann et al. demonstrated the general feasibility of the MoBI concept and provided evidence that brain dynamics can be analyzed during motion, despite massive electromyographic activity from neck and cranial muscles, as well as electrooculographic activity accompanying participants' movements (Gramann et al., 2010a). We analyzed the brain dynamics of participants attending to a stream of visual stimuli during standing, slow treadmill walking, and fast treadmill walking. The task was to respond whenever a target was detected in a stream of non-target stimuli. The onset of rare targets ('oddballs') was associated with the so-called 'oddball P3' in the event-related potential; a positive-going peak of the stimulus-locked and averaged spontaneous EEG with a latency around $300 \mathrm{~ms}$, that accompanies infrequent and task-relevant stimuli (Sutton et al., 1965; Picton, 1992). The P3 is thought to reflect stimulus updating and categorization processes being active when stimulus detection engages memory operations (Polich, 2007).

However, this component of the event-related potential was visible only after dissociating and filtering out activity related to horizontal and vertical eye movement and activity arising from neck, supracranial, and facial muscles. Analyses of sensor based data without spatially filtering would have left the P300 buried under activity arising from these nonbrain processes while participants were actively moving (Kerick et al., 2009). Increasing non-brain activity with increasing walking speed, however, revealed the 'cognitive nature' of eye movement and muscle activity. While not measured in our study, prior studies have demonstrated that increasing walking speed increases head deviations in all three movement planes (up-down, front-back, left-right). Therefore, to detect and discriminate target stimuli, which appeared on a stationary monitor in front of the subjects, participants had to compensate for displacements of the head. This was done by activating the appropriate neck muscles and moving the eyes so as to retain focus on the center of the screen (Hirasaki et al., 1999; Mulavara et al., 2002) (Figure 2A).

Back-projecting only the activity of IC processes with equivalent dipole models located in the brain (i.e., by filtering out any nonbrain related activity of the decomposed signal), revealed significantly increased P3 amplitudes for target as compared to nontarget stimuli (Figure 2B). More importantly, P3 amplitudes for target stimuli did not differ irrespective of 

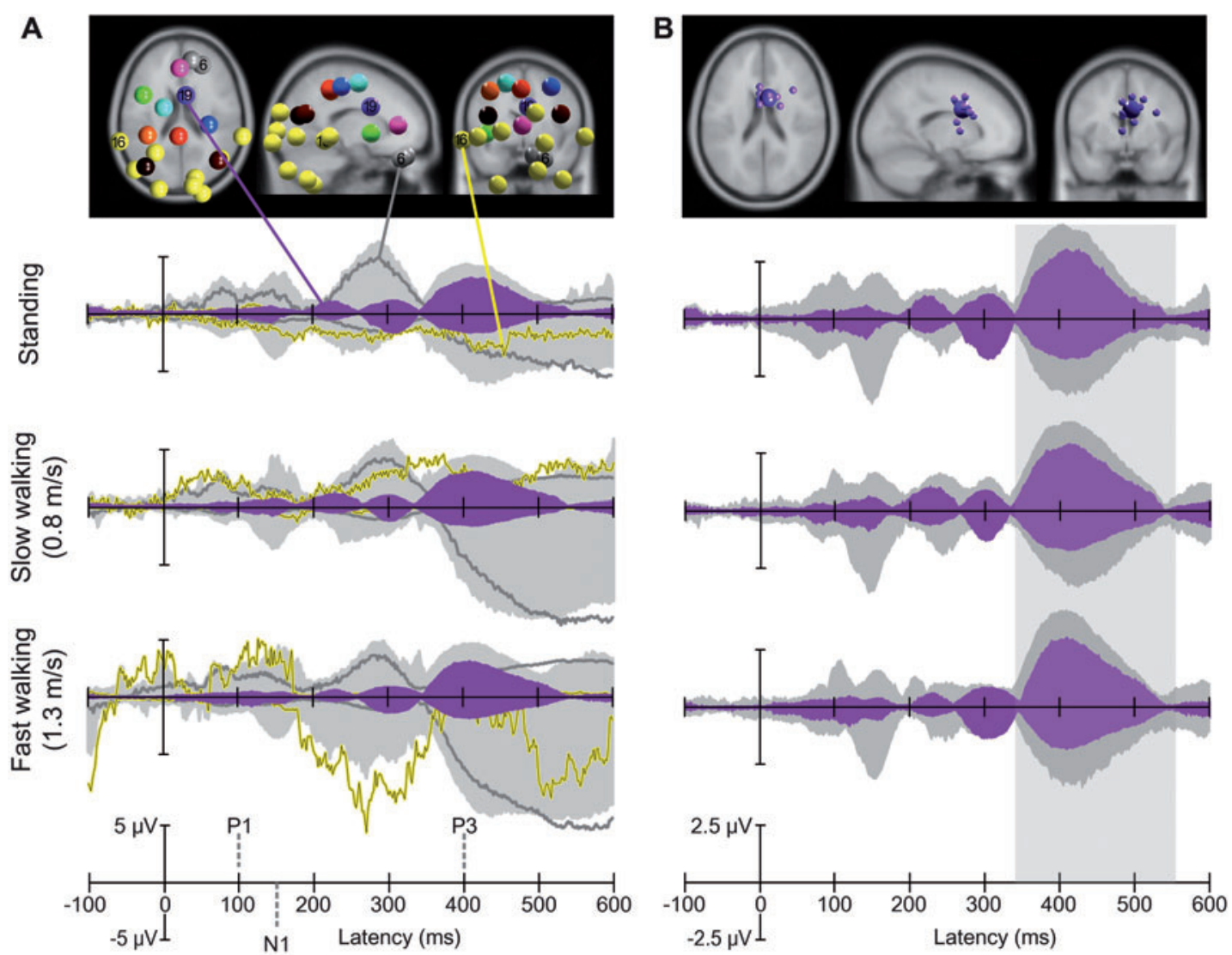

Figure 2 Visual target stimulus responses in three movement conditions. (A) Centroids of IC clusters (colored spheres) visualized in the MNI brain volume in horizontal, sagittal, and coronal views. Gray and yellow spheres represent eye and neck muscle activity clusters, respectively. Other-colored spheres mark centroids of brain IC clusters. Grand-mean event related potential (ERP) envelopes (maximum and minimum channel ERP values at each latency) time-lockedto target stimuli onset are shown below the IC cluster visualization for three different movement conditions. The light gray shaded area shows the summed back-projection of all IC clusters. The purple shaded area shows the backprojected contribution of the IC cluster with equivalent dipoles in or near anterior cingulate cortex (19). Yellow traces and dark gray traces show the back-projected contribution of a representative neck muscle cluster (16) and a representative eye movement cluster (6), respectively. (B) Centroid (bigger sphere) and individual ICs (smaller spheres) of the IC cluster contributing most strongly to the P3 peak (Cluster 19 in A). This cluster contained 13 ICs from 11 subjects. The ICs were located in or near anterior cingulate cortex (BA32). Grand-mean ERP envelopes (time-locked to target stimuli onset) are shown for the back-projected contribution of the IC cluster (purple shaded area, as in A but note the change in vertical axis scale) and for the summed back-projection of IC clusters within the gray matter of the brain (gray shaded area, different than A, excludes eye and muscle artifact ICs). The latency range of the P300 response is highlighted in light gray.

whether the participants were standing, walking slowly, or walking more quickly. This study demonstrated the viability of recording brain activity accompanying cognitive processes during whole body movement.

\section{Artifact pre-processing for extensive movements} Although ICA can remove many artifacts from EEG signals, there are times when additional signal processing is necessary. When the head undergoes particularly rapid movements, such as during running, mechanical artifacts can dominate the signal. Gwin and colleagues (Gwin et al., 2010) recently applied an artifact template regression procedure to remove running related movement activity from EEG signals. This procedure was based on existing artifact removal techniques, designed for time-invariant noise sources (Allen et al., 2000). To address the time-varying nature of gait related movement artifact, the authors used stride time-warping prior to applying the artifact template regression.

The efficacy of this technique was tested on EEG collected during treadmill running, a relatively rhythmic motor task. However, such an artifact removal procedure is not inherently limited to removing quasi-rhythmic motor artifacts. It may be possible to use a related procedure to remove movement artifact from signals recorded during more complex movements, such as rapid directional changes, or from signals recorded in harsher environments, such as during automotive transport over rough terrain, provided enough trials are available for the creation of an appropriate set of artifact templates and movement related kinematic signals are available for performing appropriate time-warping. Successful recording of EEG under these conditions remains to be seen. 


\section{Conclusion}

Animal studies using mobile imaging approaches demonstrate new insights into the brain dynamics of actively behaving animals. The modulation of brain dynamics by behavioral states in these studies complements the existing view of human brain function, as investigated using traditional imaging methods like fMRI or MEG. Future studies, using a mobile imaging approach, will have to overcome the restrictions associated with immobile sensor arrays and immobile attitudes towards analyzes of EEG signals. Making use of fast developing sensor technologies, growing computational power, and newly available analyzes techniques more data streams can be recorded and analyzed in naturally behaving humans including high density EEG, peripheral physiological activity, high definition visual scene recordings (including eye movements), and whole body kinematics. This approach to investigating the human brain in action opens new avenues in systemic human neuroscience.

\section{Acknowledgments}

This work was supported by a gift from the Swartz Foundation (Old Field, NY) and by grants from the National Science Foundation, USA (NSF IIS-0613595, TDLC-0542013), the Aiming for the Top University Plan of National Chiao Tung University, the Ministry of Education, Taiwan, the UST-UCSD International Center of Excellence in Advanced Bio-engineering sponsored by the Taiwan National Science Council I-RiCE Program under Grant Number NSC-99-2911-I-009-101, and the Army Research Laboratory under Cooperative Agreement Number W911NF-10-2-0022. The views and the conclusions contained in this document are those of the authors and should not be interpreted as representing the official policies, either expressed or implied, of the Army Research Laboratory or the US Government. The US Government is authorized to reproduce and distribute reprints for government purposes notwithstanding any copyright notation herein.

\section{References}

Aguirre, G.K., Zarahn, E., and D’Esposito, M. (1998). Neural components of topographical representation. Proc. Natl. Acad. Sci. USA 95, 839-846.

Allen, P.J., Josephs, O., and Turner, R. (2000). A method for removing imaging artifact from continuous EEG recorded during functional MRI. NeuroImage 12, 230-239.

Allum, J.H.J., Carpenter, M.G., and Adkin, A.L. (2001). Balance control analysis as a method for screening and identifying balance deficits. Vestibular Labyrinth in Health and Disease 942, 413-427.

Anderson, M.L. (2003). Embodied cognition: a field guide. Artif. Intell. 149, 91-130.

Aniss, A.M., Gandevia, S.C., and Burke, D. (1992). Reflex responses in active muscles elicited by stimulation of low-threshold afferents from the human foot. J. Neurophysiol. 67, 1375-1384.

Gruetzmann, A., Hansen, S., and Mueller, J. (2007). Novel dry electrodes for ECG monitoring. Physiol. Meas. 28, 1375.

Baek, J.-Y., An, J.-H., Choi, J.-M., Park, K.-S., and Lee, S.-H. (2008). Flexible polymeric dry electrodes for the long-term monitoring of ECG. Sensor. Actuat A-Phys. 143, 423-429.
Bakker, M., Verstappen, C.C.P., Bloem, B.R., and Toni, I. (2007). Recent advances in functional neuroimaging of gait. J. Neural. Transm. 114, 1323-1331.

Bardy, B.G., Warren, W.H., and Kay, B.A. (1996). Motion parallax is used to control postural sway during walking. Exp. Brain. Res. 111, 271-282.

Beckmann, L., Neuhaus, C., Medrano, G., Jungbecker, N., Walter, M., Gries, T., and Leonhardt, S. (2010). Characterization of textile electrodes and conductors using standardized measurement setups. Physiol. Meas. 31, 233.

Beloozerova, I.N., Zelenin, P.V., Popova, L.B., Orlovsky, G.N., Grillner, S., and Deliagina, T.G. (2003). Postural control in the rabbit maintaining balance on the tilting platform. J. Neurophysiol. 90, 3783-3793.

Beloozerova, I.N., Sirota, M.G., Orlovsky, G.N., and Deliagina, T.G. (2005). Activity of pyramidal tract neurons in the cat during postural corrections. J. Neurophysiol. 93, 1831-1844.

Berthoz, A., Lacour, M., Soechting, J.F., and Vidal, P.P. (1979). The role of vision in the control of posture during linear motion. Prog. Brain Res. 50, 197-209.

Biguer, B., Donaldson, I.M., Hein, A., and Jeannerod, M. (1988). Neck muscle vibration modifies the representation of visual motion and direction in man. Brain 111, 1405-1424.

Bove, M., Courtine, G., and Schieppati, M. (2002). Neck muscle vibration and spatial orientation during stepping in place in humans. J. Neurophysiol. 88, 2232-2241.

Boyd, L.A., Vidoni, E.D., and Daly, J.J. (2007). Answering the call: the influence of neuroimaging and electrophysiological evidence on rehabilitation. Phys. Ther. 87, 684-703.

Bronstein, A.M., Hood, J.D., Gresty, M.A., and Panagi, C. (1990). Visual control of balance in cerebellar and Parkinsonian syndromes. Brain 113, 767-779.

Bull, M.L., Vitti, M., and Defreitas, V. (1985). Electromyographic study of the trapezius (upper portion) and levator scapulae muscles in some movements of the shoulders. Anat. Anzeiger. 159, 21-27.

Caldwell, J.A., Prazinko, B.F., and Hall, K.K. (2000). The effects of body posture on resting electroencephalographic activity in sleep-deprived subjects. Clin. Neurophysiol. 111, 464470.

Caldwell, J.A., Prazinko, B., and Caldwell, J.L. (2003). Body posture affects electroencephalographic activity and psychomotor vigilance task performance in sleep-deprived subjects. Clin. Neurophysiol. 114, 23-31.

Capaday, C., Lavoie, B.A., Barbeau, H., Schneider, C., and Bonnard, M. (1999). Studies on the corticospinal control of human walking. I. Responses to focal transcranial magnetic stimulation of the motor cortex. J. Neurophysiol. 81, 129-139.

Carpenter, M.G., Frank, J.S., Silcher, C.P., and Peysar, G.W. (2001). The influence of postural threat on the control of upright stance. Exp. Brain. Res. 138, 210-218.

Casasanto, D. and Dijkstra, K. (2010). Motor action and emotional memory. Cognition 115, 179-185.

Chi, Y.M. and Cauwenberghs, G. (2010). Wireless non-contact EEG/ECG electrodes for body sensor networks. Proc. Int. Conf. Body Sensor Networks (BSN), June 7-9, 2010, Singapore, pp. 297-301.

Chi, Y.M., Deiss, S.R., and Cauwenberghs, G. (2009). Noncontact low power EEG/ECG electrode for high density wearable biopotential sensor networks. Wearable and Implantable Body Sensor Networks (BSN), June 3-5, 2009, Berkeley, CA, pp. 246-250. 
Chi, Y.M., Jung, T.P., and Cauwenberghs, G. (2010). Dry-contact and noncontact biopotential electrodes: methodological review. IEEE Reviews-Biomed. Eng. 3, 106-119.

Christensen, L.O.D., Andersen, J.B., Sinkjaeer, T., and Nielsen, J. (2001). Transcranial magnetic stimulation and stretch reflexes in the tibialis anterior muscle during human walking. J. Physiol. London 531, 545-557.

Cisek, P. and Kalaska, J.F. (2010). Neural mechanisms for interacting with a world full of action choices. Ann. Rev. Neurosci. 33, 269-298.

Cole, R.J. (1989). Postural baroreflex stimuli may affect EEG arousal and sleep in humans. J. Appl. Physiol. 67, 2369-2375.

Crowley, K., Sliney, A., Pitt, I., and Murphy, D. (2010). Evaluating a brain-computer interface to categorise human emotional response. advanced learning technologies (ICALT), 2010 IEEE 10th International Conference, pp. 276-278.

Daly, J.J. and Wolpaw, J.R. (2008). Brain-computer interfaces in neurological rehabilitation. Lancet Neurol. 7, 1032-1043.

Delorme, A. and Makeig, S. (2004). EEGLAB: an open source toolbox for analysis of single-trial EEG dynamics including independent component analysis. J. Neurosci. Methods 134, 9-21.

Delorme, A., Mullen, T., Kothe, C., Acar, Z.A., Bigdely-Shamlo, N., Vankov, A., and Makeig, S. (2011). EEGLAB, SIFT, NFT, BCILAB, and ERICA: new tools for advanced EEG processing. Computat. Intelligence Neurosci. 130714, doi: 10.1155/2011/ 130714.

Diener, H.C., Ackermann, H., Dichgans, J., and Guschlbauer, B. (1985). Medium- and long-latency responses to displacements of the ankle joint in patients with spinal and central lesions. Electroencephalogr. Clin. Neurophysiol. 60, 407-416.

Dietz, V. (2003). Spinal cord pattern generators for locomotion. Clin. Neurophysiol. 114, 1379-1389.

Dietz, V., Colombo, G., Jensen, L., and Baumgartner, L. (1995). Locomotor capacity of spinal cord in paraplegic patients. Ann. Neurol. 37, 574-582.

Dietz, V. and Duysens, J. (2000). Significance of load receptor input during locomotion: a review. Gait Posture 11, 102-110.

Dijkstra, T.M.H., Schoner, G., and Gielen, C.C.A.M. (1994). Temporal stability of the action-perception cycle for postural control in a moving visual environment. Exp. Brain Res. 97, 477-486.

Dobkin, B.H., Firestine, A., West, M., Saremi, K., and Woods, R. (2004). Ankle dorsiflexion as an fMRI paradigm to assay motor control for walking during rehabilitation. NeuroImage 23, 370-381.

Doyon, J., Song, A.W., Karni, A., Lalonde, F., Adams, M.M., and Ungerleider, L.G. (2002). Experience-dependent changes in cerebellar contributions to motor sequence learning. Proc. Natl. Acad. Sci. USA 99, 1017-1022.

Drew, T., Prentice, S., and Schepens, B. (2004). Cortical and brainstem control of locomotion. Prog. Brain Res. 143, 251-261.

Elbert, T., Pantev, C., Wienbruch, C., Rockstroh, B., and Taub, E. (1995). Increased cortical representation of the fingers of the left hand in string players. Science 270, 305-307.

Elble, R.J. and Leffler, K. (2000). Pushing and pulling with the upper extremities while standing: the effects of mild Alzheimer dementia and Parkinson's disease. Movement Disord. 15, 255268.

Eric, W.S., Peter, T., William, A.S., Tobin, M., Theresa, M.V., and Robert, M. (2009). A Novel Dry Electrode for Brain-Computer Interface. In: Proceedings of the 13th International Conference on Human-Computer Interaction. Part II: Novel Interaction Methods and Techniques. (San Diego, CA: Springer-Verlag), pp. 623-631.
Farrell, M.J. and Robertson, I.H. (1998). Mental rotation and the automatic updating of body-centered spatial relationships. J. Exp. Psychol. Learn 24, 227-233.

Ferree, T.C., Russell, G.S., and Tucker, D.M. (2001). Scalp electrode impedance, infection risk, and EEG data quality. Clinical Neurophysiology 112, 536-544.

Ferris, D.P., Gordon, K.E., Beres-Jones, J.A., and Harkema, S.J. (2004). Muscle activation during unilateral stepping occurs in the nonstepping limb of humans with clinically complete spinal cord injury. Spinal Cord 42, 14-23.

Fitzsimmons, N.A., Lebedev, M.A., Peikon, I.D., and Nicolelis, M.A. (2009). Extracting kinematic parameters for monkey bipedal walking from cortical neuronal ensemble activity. Front. Integr. Neurosci. 3, 3.

Fong, A.J., Roy, R.R., Ichiyama, R.M., Lavrov, I., Courtine, G., Gerasimenko, Y., Tai, Y.C., Burdick, J., and Edgerton, V.R. (2009). Recovery of control of posture and locomotion after a spinal cord injury: solutions staring us in the face. Prog. Brain Res. 175, 393-418.

Fonseca, C., Cunha, J.S., Martins, R., Ferreira, V., Sá, J.M.d., Barbosa, M., and Silva, A.M.d. (2007). A novel dry active electrode for EEG recording. IEEE T Bio-med. Eng. 54, 162-165.

Fox, W.L. (1984). Dandy of Johns Hopkins (Baltimore, MD: Williams and Wilkins).

Gramann, K. (in press). Embodiment of spatial reference frames and individual differences in reference frame proclivity. Spatial Cognition and Computation. DOI: 10.1080/13875868.2011. 589038.

Gramann, K., Muller, H.J., Eick, E.M., and Schonebeck, B. (2005). Evidence of separable spatial representations in a virtual navigation task. J. Exp. Psychol. Human 31, 1199-1223.

Gramann, K., Muller, H.J., Schonebeck, B., and Debus, G. (2006). The neural basis of ego- and allocentric reference frames in spatial navigation: evidence from spatio-temporal coupled current density reconstruction. Brain Res. 1118, 116-129.

Gramann, K., Gwin, J.T., Bigdely-Shamlo, N., Ferris, D.P., and Makeig, S. (2010a). Visual evoked responses during standing and walking. Front. Hum. Neurosci. 5, 12.

Gramann, K., Onton, J., Riccobon, D., Mueller, H.J., Bardins, S., and Makeig, S. (2010b). Human brain dynamics accompanying use of egocentric and allocentric reference frames during navigation. J. Cognitive Neurosci. 22, 2836-2849.

Grillner, S., Wallen, P., Saitoh, K., Kozlov, A., and Robertson, B. (2008). Neural bases of goal-directed locomotion in vertebrates - an overview. Brain Res. Rev. 57, 2-12.

Griss, P., Enoksson, P., Tolvanen-Laakso, H.K., Meriläinen, P., Ollmar, S., and Stemme, G. (2001). Micromachined electrodes for biopotential measurement. IEEE J. Microelectromechanical Systems 10, 10-15.

Griss, P., Tolvanen-Laakso, H.K., Meriläinen, P., and Stemme, G. (2002). Characterization of micromachined spiked biopotentials electrodes. IEEE T Bio-med Eng. 49, 597-604.

Gron, G., Wunderlich, A.P., Spitzer, M., Tomczak, R., and Riepe, M.W. (2000). Brain activation during human navigation: genderdifferent neural networks as substrate of performance. Nat. Neurosci. 3, 404-408.

Gwin, J.T., Gramann, K., Makeig, S., and Ferris, D.P. (2010). Removal of movement artifact from high-density EEG recorded during walking and running. J. Neurophysiol. 103, 3526-3534.

Gwin, J.T., Gramann, K., Makeig, S., and Ferris, D.P. (2011). Electrocortical activity is coupled to gait cycle phase during treadmill walking. NeuroImage 54, 1289-1296.

Harada, T., Miyai, I., Suzuki, M., and Kubota, K. (2009). Gait capacity affects cortical activation patterns related to speed control 
in the elderly. Experimental brain research. Experimentelle Hirnforschung. Experimentation Cerebrale 193, 445-454.

Hatsopoulos, N.G. and Donoghue, J.P. (2009). The science of neural interface systems. Annu. Rev. Neurosci. 32, 249-266.

Hauer, K., Pfisterer, M., Weber, C., Wezler, N., Kliegel, M., and Oster, P. (2003). Cognitive impairment decreases postural control during dual tasks in geriatric patients with a history of severe falls. J. Am. Geriatr. Soc. 51, 1638-1644.

Hayhoe, M. and Ballard, D. (2005). Eye movements in natural behavior. Trend. Cogn. Sci. 9, 188-194.

Heuninckx, S., Wenderoth, N., Debaere, F., Peeters, R., and Swinnen, S.P. (2005). Neural basis of aging: the penetration of cognition into action control. J. Neurosci. 25, 6787-6796.

Heuninckx, S., Wenderoth, N., and Swinnen, S.P. (2008). Systems neuroplasticity in the aging brain: recruiting additional neural resources for successful motor performance in elderly persons. J. Neurosci. 28, 91-99.

Hiraoka, K. and Abe, K. (2007). Cortical and spinal control of ankle joint muscles before and during gait initiation. Somatosens. Mot. Res., 24 127-133.

Hirasaki, E., Moore, S.T., Raphan, T., and Cohen, B. (1999). Effects of walking velocity on vertical head and body movements during locomotion. Exp. Brain Res. 127, 117-130.

Hlavacka, F., Mergner, T., and Krizkova, M. (1996). Control of the body vertical by vestibular and proprioceptive inputs. Brain Res. Bull. 40, 431-434.

Hoffmann, K.P. and Ruff, R. (2007). Flexible dry surface-electrodes for ECG long-term monitoring. EMBS 2007. In: 29th Annual International Conference of the IEEE, pp. 5739-5742.

Horak, F.B. and Diener, H.C. (1994). Cerebellar control of postural scaling and central set in stance. J. Neurophysiol. 72, 479-493.

Horak, F.B. and Nashner, L.M. (1986). Central programming of postural movements-adaptation to altered support-surface configurations. J. Neurophysiol. 55, 1369-1381.

Hunter, M.C. and Hoffman, M.A. (2001). Postural control: visual and cognitive manipulations. Gait Posture 13, 41-48.

Iglesias, C., Nielsen, J.B., and Marchand-Pauvert, V. (2008). Corticospinal inhibition of transmission in propriospinal-like neurones during human walking. Eur. J. Neurosci. 28, 1351-1361.

Inglis, J.T., Horak, F.B., Shupert, C.L., and Jonesrycewicz, C. (1994). The importance of somatosensory information in triggering and scaling automatic postural responses in humans. Exp. Brain Res. $101,159-164$.

Ionta, S., Ferretti, A., Merla, A., Tartaro, A., and Romani, G.L. (2010). Step-by-step: the effects of physical practice on the neural correlates of locomotion imagery revealed by fMRI. Hum. Brain Mapp. 31, 694-702.

Irani, F., Platek, S.M., Bunce, S., Ruocco, A.C., and Chute, D. (2007). Functional near infrared spectroscopy (fNIRS): an emerging neuroimaging technology with important applications for the study of brain disorders. Clin. Neuropsychol. 21, 9-37.

Jacobs, J.V. and Horak, F.B. (2007). Cortical control of postural responses. J. Neural. Transm. 114, 1339-1348.

Jahn, K., Deutschlander, A., Stephan, T., Strupp, M., Wiesmann, M., and Brandt, T. (2004). Brain activation patterns during imagined stance and locomotion in functional magnetic resonance imaging. NeuroImage 22, 1722-1731.

Jahn, K., Deutschlader, A., Stephan, T., Kalla, R., Wiesmann, M., Strupp, M., and Brandt, T. (2008a). Imaging human supraspinal locomotor centers in brainstem and cerebellum. NeuroImage 39, 786-792.

Jahn, K., Deutschlander, A., Stephan, T., Kalla, R., Hufner, K., Wagner, J., Strupp, M., and Brandt, T. (2008b). Supraspinal locomotor control in quadrupeds and humans. Using eye movements as an experimental probe of brain function-a Symposium in Honor of Jean Buttner-Ennever 171, 353-362.

Jahn, K., Wagner, J., Deutschlander, A., Kalla, R., Hufner, K., Stephan, T., Strupp, M., and Brandt, T. (2009). Human hippocampal activation during stance and locomotion fMRI study on healthy, blind, and vestibular-loss subjects. Ann. Ny. Acad. Sci. 1164, 229-235.

Jahn, K. and Zwergal, A. (2010). Imaging supraspinal locomotor control in balance disorders. Restor. Neurol. Neuros. 28, 105-114.

Jeka, J., Oie, K.S., and Kiemel, T. (2000). Multisensory information for human postural control: integrating touch and vision. Exp. Brain Res. 134, 107-125.

Jeka, J.J., Allison, L.K., and Kiemel, T. (2010). The dynamics of visual reweighting in healthy and fall-prone older adults. J Motor Behav 42, 197-208.

Johansson, R., Magnusson, M., and Fransson, P.A. (1995). Galvanic vestibular stimulation for analysis of postural adaptation and stability. IEEE T Bio-med. Eng. 42, 282-292.

Kavounoudias, A., Roll, R., and Roll, J.P. (2001). Foot sole and ankle muscle inputs contribute jointly to human erect posture regulation. J. Physiol-London 532, 869-878.

Kelly, A.M.C. and Garavan, H. (2005). Human functional neuroimaging of brain changes associated with practice. Cereb. Cortex. 15, 1089-1102.

Kempermann, G., Fabel, K., Ehninger, D., Babu, H., Leal-Galicia, P., Garthe, A., and Wolf, S.A. (2010). Why and how physical activity promotes experience-induced brain plasticity. Front. Neurosci. 4, 189.

Kerick, S.E., Oie, S.E., and McDowell, K. (2009). Assessment of EEG signal quality in motion environments. Aberdeen Proving Ground, MD: US Army Research Laboratory. Report No.: ARLTN-355.

Kiemel, T., Oie, K.S., and Jeka, J.J. (2006). Slow dynamics of postural sway are in the feedback loop. J. Neurophysiol. 95, 1410-1418.

Kim, H.K., Park, S., and Srinivasan, M.A. (2009). Developments in brain-machine interfaces from the perspective of robotics. Hum. Movement Sci. 28, 191-203.

Ko, L.-W., Chiou, J.-C., Lin, C.-T., Hong, C.-T., Jung, T.-P., Liang, S.-F., and Jeng, J.-L. (2006). Using novel MEMS EEG sensors in detecting drowsiness application. In: Proceeding of IEEE Biomedical Circuits and Systems Conference (BioCAS 2006), pp. 33-36.

la Fougere, C., Zwergal, A., Rominger, A., Forster, S., Fesl, G., Dieterich, M., Brandt, T., Strupp, M., Bartenstein, P., and Jahn, K. (2010). Real versus imagined locomotion: a [18F]-FDG PETfMRI comparison. NeuroImage 50, 1589-1598.

Leuthardt, E.C., Schalk, G., Roland, J., Rouse, A., and Moran, D.W. (2009). Evolution of brain-computer interfaces: going beyond classic motor physiology. Neurosurg. Focus 27, E4.

Lewko, J.P. (1996). Assessment of muscle electrical activity in spinal cord injury subjects during quiet standing. Paraplegia 34 , 158-163.

Liao, L.-D., Wang, I.-J., Chen, S.-F., Chang, J.-Y., and Lin, C.-T. (2011). Design, fabrication and experimental validation of a novel dry contact sensor for measuring electroencephalography signals without skin preparation. Sensors 11, 5819-5834.

Lin, C.-T., Ko, L.-W., Chiou, J.-C., Duann, J.-R., Huang, R.-S., Chiu, T.-W., Liang, S.-F., and Jung, T.-P. (2008). Noninvasive neural prostheses using mobile and wireless EEG. P. IEEE 96, 1167-1183.

Lin, C.T., Liao, L.D., Liu, Y.H., Wang, I.J., Lin, B.S., and Chang, J.Y. (2011). Novel dry polymer foam electrodes for long-term EEG Measurement. IEEE Bio-med. Eng. 58, 1200-1207. 
Logothetis, N.K., Pauls, J., Augath, M., Trinath, T., and Oeltermann, A. (2001). Neurophysiological investigation of the basis of the fMRI signal. Nature 412, 150-157.

Luft, A.R., Smith, G.V., Forrester, L., Whitall, J., Macko, R.F., Hauser, T.K., Goldberg, A.P., and Hanley, D.F. (2002). Comparing brain activation associated with isolated upper and lower limb movement across corresponding joints. Hum. Brain. Mapp. 17, 131-140.

Magnus, R. (1926). Some results of studies in the physiology of posture. Lancet $i i, 531-536$.

Maguire, E.A., Burgess, N., Donnett, J.G., Frackowiak, R.S., Frith, C.D., and O'Keefe, J. (1998). Knowing where and getting there: a human navigation network. Science 280, 921-924.

Maimon, G., Straw, A.D., and Dickinson, M.H. (2010). Active flight increases the gain of visual motion processing in Drosophila. Nat. Neurosci. 13, 393-399.

Makeig, S., Westerfield, M., Jung, T.P., Enghoff, S., Townsend, J., Courchesne, E., and Sejnowski, T.J. (2002). Dynamic brain sources of visual evoked responses. Science 295, 690-694.

Makeig, S., Delorme, A., Westerfield, M., Jung, T.-P., Townsend, J., Courchesne, E., and Sejnowski, T.J. (2004). Electroencephalographic brain dynamics following manually responded visual targets. PLoS Biology 2, e176.

Makeig, S., Gramann, K., Jung, T.P., Sejnowski, T.J., and Poizner, H. (2009). Linking brain, mind and behavior. Int. J. Psychophysiol. $73,95-100$.

Maki, B.E., McIlroy, W.E., and Fernie, G.R. (2003). Change-insupport reactions for balance recovery. IEEE Eng. Med. Biol. 22, 20-26.

Matthews, R., McDonald, N.J., Fridman, I., Hervieux, P., and Nielsen, T. (2005). The invisible electrode - zero prep time, ultra low capacitive sensing. 11th International Conference on Human Computer Interaction (HCII), Las Vegas, NV, pp. 22-27.

Matthews, R., McDonald, N.J., Anumula, H., Woodward, J., Turner, P.J., Steindorf, M.A., Chang, K., and Pendleton, J.M. (2007). Novel hybrid bioelectrodes for ambulatory zero-prep EEG measurements using multi-channel wireless EEG system. Lect. Notes Comput Sc. 4565, 137-146.

Matthews, R., Turner, P.J., McDonald, N.J., Ermolaev, K., Manus, T.M., Shelby, R.A., and Steindorf, M. (2008). Real time workload classification from an ambulatory wireless EEG system using hybrid EEG electrodes. EMBS 2008, 30th Annual International Conference of the IEEE, pp. 5871-5875.

MayouxBenhamou, M.A., Revel, M., and Vallee, C. (1997). Selective electromyography of dorsal neck muscles in humans. Exp. Brain. Res. 113, 353-360.

Mehta, J.P., Verber, M.D., Wieser, J.A., Schmit, B.D., and SchindlerIvens, S.M. (2009). A novel technique for examining human brain activity associated with pedaling using fMRI. J. Neurosci. Meth. 179, 230-239.

Mergner, T., Maurer, C., and Peterka, R.J. (2003). A multisensory posture control model of human upright stance. Neural Control of Space Coding and Action Production 142, 189-199.

Millan, J.D., Ferrez, P.W., Galan, F., Lew, E., and Chavarriaga, R. (2008). Non-invasive brain-machine interaction. Int. J. Pattern Recogn. 22, 959-972.

Miyai, I., Tanabe, H.C., Sase, I., Eda, H., Oda, I., Konishi, I., Tsunazawa, Y., Suzuki, T., Yanagida, T., and Kubota, K. (2001). Cortical mapping of gait in humans: a near-infrared spectroscopic topography study. NeuroImage 14, 1186-1192.

Morasso, P.G., Baratto, L., Capra, R., and Spada, G. (1999). Internal models in the control of posture. Neural Networks 12, 1173-1180.

Mulavara, A.P., Verstraete, M.C., and Bloomberg, J.J. (2002). Modulation of head movement control in humans during treadmill walking. Gait Posture 16, 271-282.
Murphy, K.R., Parasuraman, B., Pethick, N., Miller, C.J., and Fitzpatrick, S. (2002). Greater improvement in functional health status with budesonide inhalation suspension (Pulmicort Respules (TM) versus conventional therapy in children with persistent asthma. Pediatr. Res. 51, 177A.

Niell, C.M. and Stryker, M.P. (2010). Modulation of visual responses by behavioral state in mouse visual cortex. Neuron $65,472-479$.

Nielsen, J.B. (2003). How we walk: central control of muscle activity during human walking. Neuroscientist 9, 195-204.

Nunez, P.L. (1981). Electric fields of the brain: the neurophysics of EEG. (Oxford, UK: Oxford University Press).

Oie, K.S., Kiemel, T., and Jeka, J.J. (2002). Multisensory fusion: simultaneous re-weighting of vision and touch for the control of human posture. Cognitive Brain Res. 14, 164-176.

Perennou, D.A., Leblond, C., Amblard, B., Micallef, J.P., Rouget, E., and Pelissier, J. (2000). The polymodal sensory cortex is crucial for controlling lateral postural stability: evidence from stroke patients. Brain Res. Bull. 53, 359-365.

Peterka, R.J. (2002). Sensorimotor integration in human postural control. J. Neurophysiol. 88, 1097-1118.

Peterson, B.W., Goldberg, J., Bilotto, G., and Fuller, J.H. (1985). Cervicocollic reflex-its dynamic properties and interaction with vestibular reflexes. J. Neurophysiol. 54, 90-109.

Pfurtscheller, G., Leeb, R., Keinrath, C., Friedman, D., Neuper, C., Guger, C., and Slaterc, M. (2006). Walking from thought. Brain Res. 1071, 145-152.

Picton, T.W. (1992). The P300 wave of the human event-related potential. J. Clin. Neurophysiol. 9, 456-479.

Plonsey, R. and Heppner, D.B. (1967). Considerations of quasistationarity in electrophysiological systems. Bull. Math. Biophys. 29, 657-664.

Polich, J. (2007). Updating p300: an integrative theory of P3a and P3b. Clin. Neurophysiol. 118, 2128-2148.

Quant, S., Adkin, A.L., Staines, W.R., Maki, B.E., and McIlroy, W.E. (2004). The effect of a concurrent cognitive task on cortical potentials evoked by unpredictable balance perturbations. BMC Neurosci. 5, 18.

Roberto, M. (2010). The electrode - skin interface and optimal detection of bioelectric signals Physiol. Meas. 10, 31 .

Rossignol, S., Dubuc, R.J., and Gossard, J.P. (2006). Dynamic sensorimotor interactions in locomotion. Physiol. Rev. 86, 89-154.

Ruffini, G., Dunne, S., Farrés, E., Marco-Pallarés, J., Ray, C., Mendoza, E., Silva, R., and Grau, C. (2006). A dry electrophysiology electrode using CNT arrays. Sensor. Actuat. A-Phys. 132, 34-41.

Ruffini, G., Dunne, S., Fuentemilla, L., Grau, C., Farr, E., MarcoPallar, J., Watts, P.C.P., and Silva, S.R.P. (2008). First human trials of a dry electrophysiology sensor using a carbon nanotube array interface. Sensor. Actuat. A-Phys. 144, 275-279.

Sahyoun, C., Floyer-Lea, A., Johansen-Berg, H., and Matthews, P.M. (2004). Towards an understanding of gait control: brain activation during the anticipation, preparation and execution of foot movements. NeuroImage 21, 568-575.

Scherberger, H. (2009). Neural control of motor prostheses. Curr. Opin. Neurobiol. 19, 629-633.

Schubert, M., Curt, A., Jensen, L., and Dietz, V. (1997). Corticospinal input in human gait: modulation of magnetically evoked motor responses. Exp. Brain Res. 115, 234-246.

Schubert, M., Curt, A., Colombo, G., Berger, W., and Dietz, V. (1999). Voluntary control of human gait: conditioning of magnetically evoked motor responses in a precision stepping task. Exp. Brain Res. 126, 583-588.

Sherrington, C.S. (1910). Flexion-reflex of the limb, crossed extension-reflex, and reflex stepping and standing. J. PhysiolLondon 40, 28-121. 
Slobounov, S., Hallett, M., Stanhope, S., and Shibasaki, H. (2005). Role of cerebral cortex in human postural control: an EEG study. Clin. Neurophysiol. 116, 315-323.

Slobounov, S., Hallett, M., Cao, C., and Newell, K. (2008). Modulation of cortical activity as a result of voluntary postural sway direction: an EEG study. Neurosci. Lett. 442, 309-313.

Slobounov, S., Cao, C., Jaiswal, N., and Newell, K.M. (2009). Neural basis of postural instability identified by VTC and EEG. Exp. Brain Res. 199, 1-16.

Song, W.G. and Giszter, S.F. (2011). Adaptation to a cortexcontrolled robot attached at the pelvis and engaged during locomotion in rats. J. Neurosci. 31, 3110-3128.

Stoffregen, T.A., Smart, L.J., Bardy, B.G., and Pagulayan, R.J. (1999). Postural stabilization of looking. J. Exp. Psychol. Human $25,1641-1658$.

Stoffregen, T.A., Pagulayan, R.J., Bardy, B.G., and Hettinger, L.J. (2000). Modulating postural control to facilitate visual performance. Hum. Movement Sci. 19, 203-220.

Sullivan, E.V., Rose, J., Rohlfing, T., and Pfefferbaum, A. (2009). Postural sway reduction in aging men and women: relation to brain structure, cognitive status, and stabilizing factors. Neurobiol. Aging 30, 793-807.

Sutton, S., Braren, M., Zubin, J., and John, E.R. (1965). Evokedpotential correlates of stimulus uncertainty. Science 150, $1187-1188$.

Suzuki, M., Miyai, I., Ono, T., Oda, I., Konishi, I., Kochiyama, T., and Kubota, K. (2004). Prefrontal and premotor cortices are involved in adapting walking and running speed on the treadmill: an optical imaging study. NeuroImage 23, 1020-1026.

Suzuki, M., Miyai, I., Ono, T., and Kubota, K. (2008). Activities in the frontal cortex and gait performance are modulated by preparation. An fNIRS study. NeuroImage 39, 600-607.

Tachtsidis, I., Elwell, C.E., Leung, T.S., Lee, C.W., Smith, M., and Delpy, D.T. (2004). Investigation of cerebral haemodynamics by near-infrared spectroscopy in young healthy volunteers reveals posture-dependent spontaneous oscillations. Physiol. Meas. 25, 437-445.

Thakor, N.V. (1999). Biopotentials and electro-physiology measurement. In: The Measurement, Instrumentation, and Sensors Handbook. (CRC Press: Boca Raton, FL).

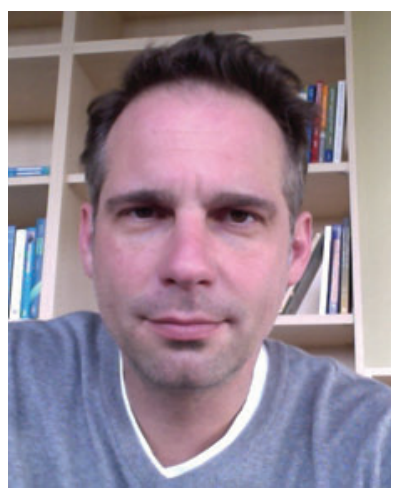

Klaus Gramann received his Pre-Diploma in Psychology from JustusLiebig-University Giessen, his Diploma and $\mathrm{PhD}$ in Psychology from RheinischWestfaelisch-Technical University Aachen in 1998 and 2002, respectively. $\mathrm{He}$ worked as a post-doctoral researcher in the Department of Psychology, LudwigMaximilians-University Munich from 2002 to 2007, and the Swartz Center for Computational Neuroscience, University of California, San Diego from 2007 to 2011. He is currently a visiting Professor at the Brain Research Center of National Chiao Tung University, Hsinchu, Taiwan. His research focuses on neural dynamics of spatial orientation and visual attention and the development of a mobile brain imaging method.
Van der Kooij, H., Jacobs, R., Koopman, B., and Grootenboer, H. (1999). A multisensory integration model of human stance control. Biol. Cybern. 80, 299-308.

Van der Kooij, H., Jacobs, R., Koopman, B., and Van der Helm, F. (2001). An adaptive model of sensory integration in a dynamic environment applied to human stance control. Biol. Cybern. 84, 103-115.

Vasavada, A.N., Peterson, B.W., and Delp, S.L. (2002). Threedimensional spatial tuning of neck muscle activation in humans. Exp. Brain Res. 147, 437-448.

Wagner, J., Stephan, T., Kalla, R., Bruckmann, H., Strupp, M., Brandt, T., and Jahn, K. (2008). Mind the bend: cerebral activations associated with mental imagery of walking along a curved path. Exp. Brain Res. 191, 247-255.

Wang, C.H., Wai, Y.Y., Kuo, B.C., Yeh, Y.Y., and Wang, J.J. (2008). Cortical control of gait in healthy humans: an fMRI study. J. Neural. Transm. 115, 1149-1158.

Willems, R.M., Hagoort, P., and Casasanto, D. (2010). Body-specific representations of action verbs: neural evidence from right- and left-handers. Psychological Science: a journal of the American Psychological Society/APS 21, 67-74.

Wilson, M. (2002). Six views on embodied cognition. Psychon. Bull. Rev. 9, 625-636.

Winter, W.R., Nunez, P.L., Ding, J., and Srinivasan, R. (2007). Comparison of the effect of volume conduction on EEG coherence with the effect of field spread on MEG coherence. Stat. Med. 26, 3946-3957.

Wirz, M., Colombo, G., and Dietz, V. (2001). Long term effects of locomotor training in spinal humans. J. Neurol. Neurosur. Ps. 71, 93-96.

Woollacott, M. and Shumway-Cook, A. (2002). Attention and the control of posture and gait: a review of an emerging area of research. Gait Posture 16, 1-14.

Xu, P.J., Zhang, H., and Tao, X.M. (2008). Textile-structured electrodes for electrocardiogram. Text. Progress 40, 183-213.

Yang, J.F. and Gorassini, M. (2006). Spinal and brain control of human walking: implications for retraining of walking. Neuroscientist 12, 379-389.

Received July 29, 2011; accepted August 10, 2011; previously published online November 10, 2011

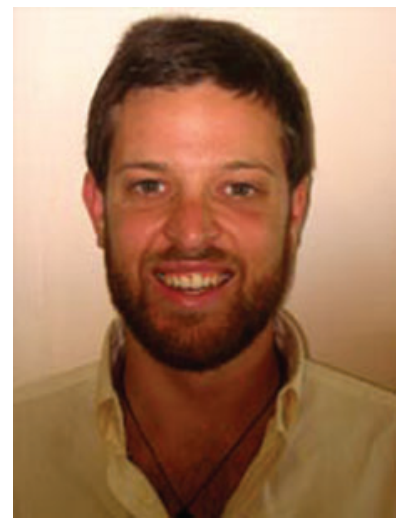

Joseph T. Gwin received his $\mathrm{BS}$ and $\mathrm{MS}$ in Engineering Sciences from Dartmouth College, Hanover, in 2006 and 2008, respectively. In 2010 he received a MS in Kinesiology from the University of Michigan, Ann Arbor, where he is currently a $\mathrm{PhD}$ candidate. He received a National Defense Science and Engineering Graduate fellowship in 2009. His current work focuses on neuromuscular biomechanics and control with a particular focus on human locomotion and novel approaches to locomotor rehabilitation in patients with neuromotor disabilities. 


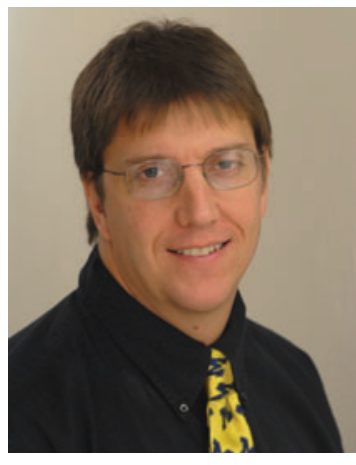

Daniel P. Ferris received his BS in Mathematics Education from the University of Central Florida in 1992, his MS in Exercise Physiology from University of Miami in 1994, and his $\mathrm{PhD}$ in Human Biodynamics from University of California, Berkeley in 1998. He worked as a post-doctoral researcher in the UCLA Department of Neurology from 1998 to 2000, and in the Department of Electrical Engineering, University of Washington 2000-2001. He is currently a Professor at the University of Michigan, Ann Arbor, in the School of Kinesiology, Department of Biomedical Engineering, and Department of Physical Medicine and Rehabilitation. He is also Associate Dean for Research in the School of Kinesiology. He studies the neuromechanical control of human locomotion in health and neurological disability. Two of his main research areas are robotic lower limb exoskeletons and mobile brain imaging during human locomotion.

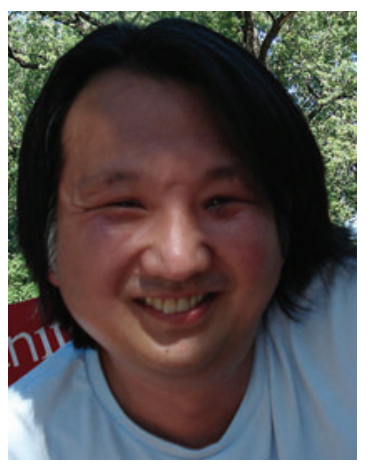

Kelvin Oie received his BS in Kinesiological Sciences in 1995, his MA in Kinesiology in 1999, and his PhD in Neuroscience and Cognitive Sciences in 2006, all from the University of Maryland in College Park, MD where he was a Graduate Research Fellow. He joined the US Army Research Laboratory in 2004 where he is currently a research scientist and program manager in the Translational Neuroscience Branch. His research has examined adaptive processes in human multisensory integration for postural control, and is currently focused on advanced approaches to measurement and understanding of human behavioral and brain dynamics in complex environments.

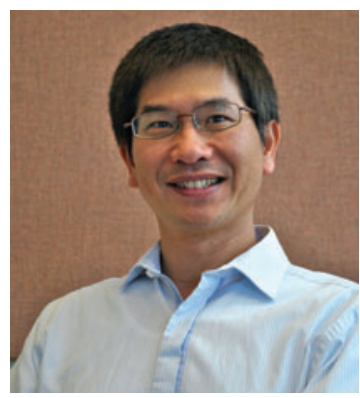

Tzyy-Ping Jung received a BS degree in Electronics Engineering from the National Chiao Tung University, Hsinchu, Taiwan, in 1984, and MS and $\mathrm{PhD}$ degrees in Electrical Engineering from The Ohio State University, Columbus, $\mathrm{OH}$, in 1989 and 1993, respectively. He was a Research Associate of the National Research Council, National Academy of Sciences, USA in 1993-1996. He is currently a Co-Director of the Center for Advanced Neurological Engineering and an Associate Director of the Swartz Center for Computational Neuroscience, University of California, San Diego. He is also an adjunct Professor of the Department of Bioengineering at UCSD and a Professor in the Department of Computer Science, National Chiao Tung University, Hsinchu, Taiwan. His research interests include areas of biomedical signal processing, cognitive neuroscience, machine learning, time-frequency analysis of human electroencephalogram, functional neuroimaging, and braincomputer interfaces and interactions.

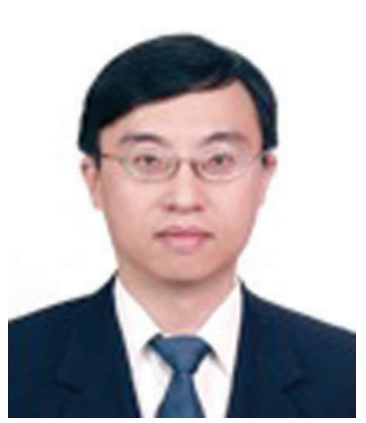

Chin-Teng Lin received his $\mathrm{PhD}$ degree in Electrical Engineering from Purdue University, West Lafayette, IN, USA in 1992. He is currently the Provost and the Chair Professor of the National Chiao-Tung University. He was elected an IEEE Fellow in 2005 for contributions to biologically inspired information systems. Dr. Lin has published over 140 journal papers, including over 80 IEEE transaction papers. His research interests are in soft computing, brain-computer interface, and cognitive neural engineering.

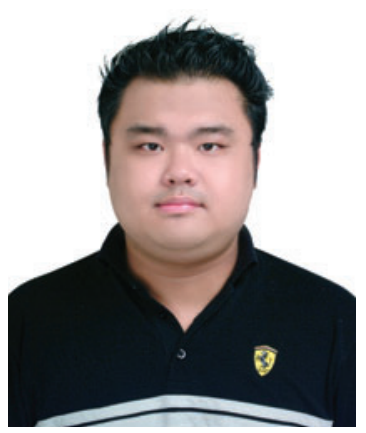

Lun-De Liao received his $\mathrm{BS}$ and MS degrees from the Department of Mechanical Engineering, Chung Yuan Christian University, Taiwan, and the Deptartment of Engineering Science from the National Cheng Kung University, Taiwan, in 2006 and 2008, respectively. He is currently studying in the $\mathrm{PhD}$ program at the Institute of Electrical Control Engineering and the Brain Research Center, National Chiao Tung University, Taiwan. His research interests include biophotonic functional imaging and advanced bio-sensing techniques.

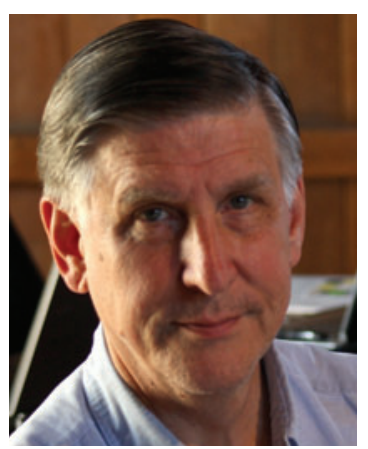

Scott Makeig earned a bachelors degree, 'Self in Experience,' at the University of California Berkeley and a PhD 'Music Psychobiology,' from the University of California San Diego. He returned to UCSD in 2002 as founding Director of the Swartz Center for Computational Neuroscience, as a Research Scientist, and is currently an Adjunct Professor of Neuroscience. His research is in the area of computational cognitive neuroscience, particularly on analysis of highdensity EEG for which he has pioneered applications of time/ frequency analysis and independent component analysis (ICA). He has defined and is now developing a novel imaging modality, Mobile Brain/Body Imaging (MoBI) combining EEG, body motion capture, eye gaze and scene video, and other information to understand the distributed EEG dynamics supporting motivated actions and interactions. 